\title{
Physalis angulata L. (Solanaceae), naturalizada en la Península Ibérica
}

\section{Pere Aymerich}

C. Barcelona, 29. 08600 Berga.

\section{Correspondencia}

Pere Aymerich

E-mail: pere_aymerich@yahoo.es

Recibido: 20 octubre 2020

Aceptado: 22 octubre 2020

Publicado on-line: 11 noviembre 2020

Editado por: Marta Recio Criado

\section{Resumen}

Se informa de la presencia de Physalis angulata como especie naturalizada en cultivos de la Península Ibérica.

Palabras clave: Flora alóctona. Solanaceae. Physalis. España. Cataluña.

\begin{abstract}
Physalis angulata L. (Solanaceae), naturalized in the Iberian Peninsula

The presence of Physalis angulata is reported in the Iberian Peninsula, as an alien species naturalized in cultivated fields.
\end{abstract}

Key words: Alien flora. Solanaceae. Physalis. Spain. Catalonia.
Physalis angulata L., Sp. Pl.: 183. 1753.

ESPAÑA, GIRONA: Colomers, área entre Els Recs y el Pla de Roda, 31TDG9758-9759, 25 m.s.n.m., campos de maiz (Zea mays), 15-X-2020, P. Aymerich s.n. (MA-01-00940623) (Fig1).

En la revisión del género Physalis L. de Flora iberica (Sanz \& Sobrino, 2012) se aceptaron cuatro especies. Una es la aparentmente autóctona $P$. alkekengi $\mathrm{L}$., que según evidencias diversas (Whitson \& Manos, 2005; Zamora-Tavares et al., 2016; Deanna et al., 2019; Rodríguez et al., 2020) es preferible incluir en el género monotípico y de distribución euroasiática Alkekengi Mill., con el nombre Alkekengi officinarum Moench. Las tres especies restantes se incluyen en el género Physalis s.str., de distribución americana, y son $P$. peruviana L., $P$. ixocarpa Brot. y $P$. philadelphica Lam. Sólo una de estas especies $(P$. ixocarpa) parece que ha establecido poblaciones naturalizadas en hábitats seminaturales del ámbito ibérico (Batard, 1970; Vázquez et al., 2002; Verloove, 2003), pero lo más habitual es que se detecten esporádicamente, con carácter de alóctonas casuales. El incremento reciente de sus citas estaría relacionado básicamente con el uso de sus frutos en repostería y con su cultivo en huertas. Una quinta especie de Physalis había sido indicada en la Península, pero pasó desapercibida en Flora iberica. Se trata de $P$. viscosa L., citada en Tarragona unas décadas antes (sub $P$. fusco-maculata De Rouville) por Casasayas (1989) y de la que existen varios pliegos en el herbario BCN recolectados en 1980-1981 (Aymerich \& Sáez, 2015). La presencia reciente de algunas poblaciones naturalizadas de $P$. viscosa está confirmada en Cataluña (Aymerich, 2016; Aymerich \& Sáez, 2019) y en el sur de Francia (Tison et al., 2014). Además de estas especies de presencia segura, también había sido citada en Iberia $P$. angulata $L$., concretamente en Portugal (Amaral Franco \& Pinto da Silva, 1964), pero se constató que se trataba de una confusión con P. ixocarpa (Batard, 1970).

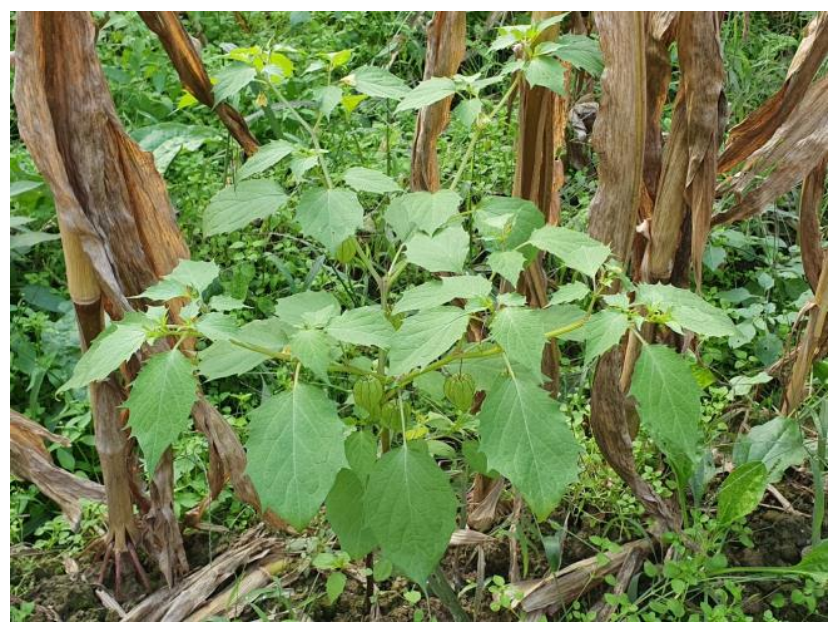

Figura 1: -Hábito de Physalis angulata.

Figure 1: Physalis angulata habit

Recientemente se ha podido constatar la aparente naturalización de $P$. angulata en la Península lbérica, en concreto en el noreste de Cataluña. Esta especie se observó en octubre de 2020 como mala hierba en campos de maíz, sobre una superficie de unas 20 ha y con abundancias muy variables según las parcelas cultivadas, desde menos de diez individuos por campo hasta cientos. La población conjunta se estimó en varios miles. Crecía en compañía de plantas frecuentes en las comunidades arvenses de verano y otoño en los campos irrigados mediterráneos, incluidas otras solanáceas (Solanum nigrum, $S$. chenopodioides, Datura stramonium). Es interesante destacar que entre estas solanáceas había unos pocos individuos de Nicandra physalodes (L.) Gaertn., especie que tan sólo cuenta con dos citas modernas en Cataluña (Casasayas, 1989; Senar \& Cardero, 2019). Considerando que $P$. angulata es una especie anual, la causa más probable de su abundancia en estos campos es la contaminación de una partida de granos de maíz con semillas de Physalis, lo que también podría explicar la aparición 
local de Nicandra. Su persistencia en esa zona en los próximos años parece casi segura, pues produce grandes cantidades de semillas que tienen una alta capacidad de germinar y de persistir viables en el suelo (Travlos, 2012; Ozaslan et al., 2017).

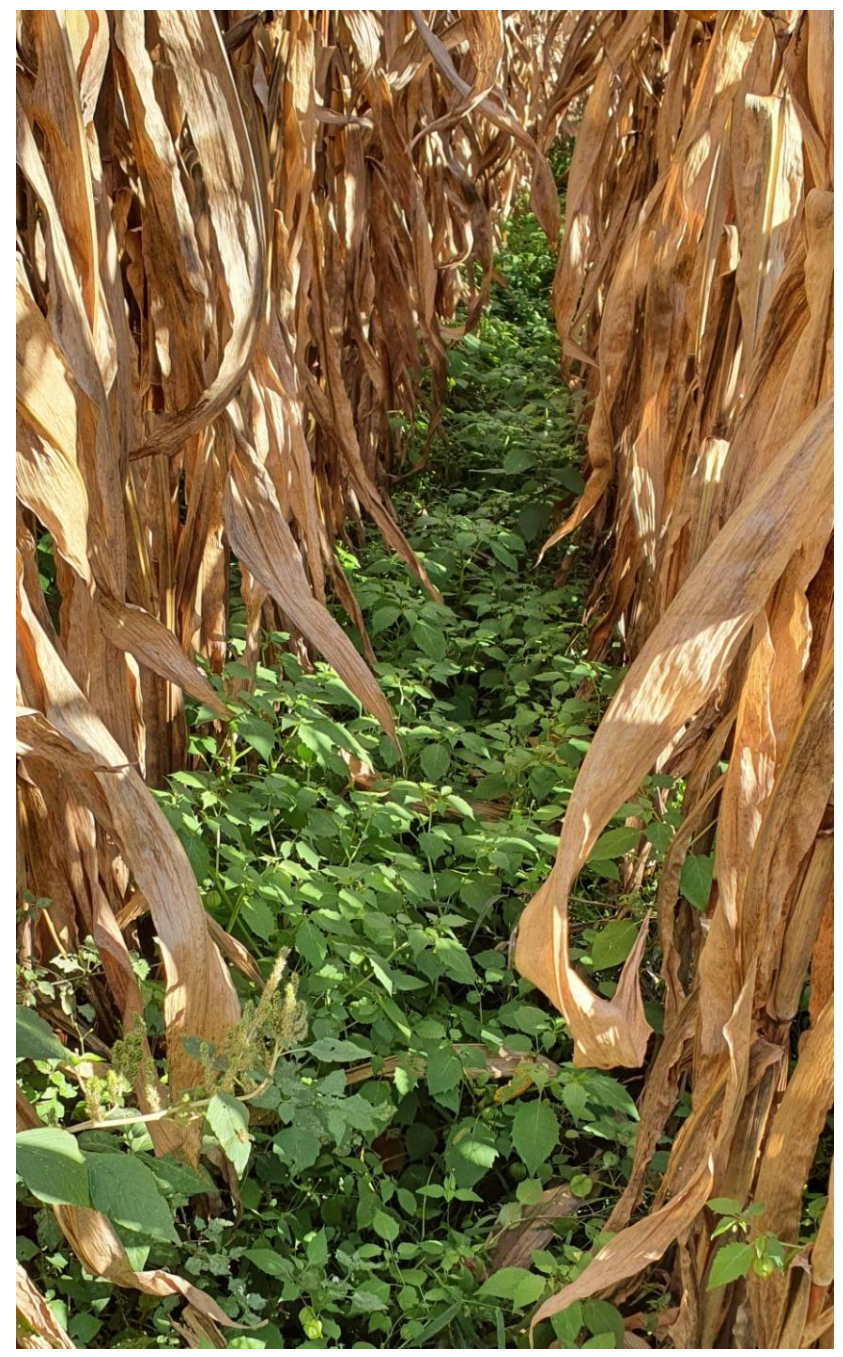

Figura 2: Población de Physalis angulata en un campo de maiz

Figure 2: Physalis angulata population in a corn field

$P$. angulata es originaria de América, donde se encuentra desde el sur de Estados Unidos hasta Argentina (Waterfall, 1958; Travlos, 2013), si bien su área nativa no es bien conocida, ya que se habría dispersado desde antiguo por vía antrópica. El cultivo tradicional a pequeña escala de esta especie, por sus frutos, está documentado por lo menos en México (Vargas-Ponce et al., 2016) y también se cultiva ocasionalmente fuera de América, por sus frutos o como ornamental (Hawkes, 1972; Stesevic \& Bubanja, 2017). En América parece tener preferencia por suelos húmedos en hábitats riparios, aunque también se encuentra en bosques claros, bordes forestales, pastos, cultivos y zonas perturbadas (Sullivan, 2004; Ward, 2008; Landrum et al., 2013). Actualmente es una especie ampliamente distribuida en las regiones subtropicales y tropicales de África, Asia y Oceanía, que colonizó en diversos periodos históricos (Travlos, 2013). En algunas zonas su llegada es antigua, como en el caso de Australia, donde ya está documentada en 1802 (Bean, 2006). En estas áreas vive especialmente en campos y otros hábitats antrópicos, pero también se puede encontrar en hábitats naturales, en particular ambientes riparios (Travlos, 2013; Electronic Flora of South Australia, 2020).

En Europa y en la zona mediterránea se ha citado en bastantes sectores, pero con grados diversos de naturalización. En general, se trata de una especie alóctona casual en el centro y oeste del continente, donde su presencia suele asociarse a escapes de cultivos 0 al transporte accidental de semillas en cargas de soja y cereales (Essl \& Rabitstch, 2002; Verloove, 2006; Stace, 2010; Pysek et al., 2012). Por el contrario, en el Mediterráneo oriental (Turquía y Grecia) se conoce como especie naturalizada infestante de cultivos desde hace un par de décadas (Gönen et al., 2000; Travlos et al., 2010) y se ha señalado también como naturalizada en Croacia (Milovic et al., 2010). Hacia el oeste, en Italia fue indicada como alóctona casual en el norte y centro (Galasso et al., 2018), si bien está en expansión y poco después se elevó su estatus a naturalizada (Berselli et al., 2019) y se amplió su distribución hasta el extremo sur (Musarella et al., 2020). Las citas del norte de Italia son, según los datos disponibles, las más próximas a la Península Ibérica. No se conocen datos publicados sobre observaciones en Francia, donde $P$, angulata no es citada en las síntesis florísticas recientes (Tison \& Foucault, 2014; Tison et al., 2014).

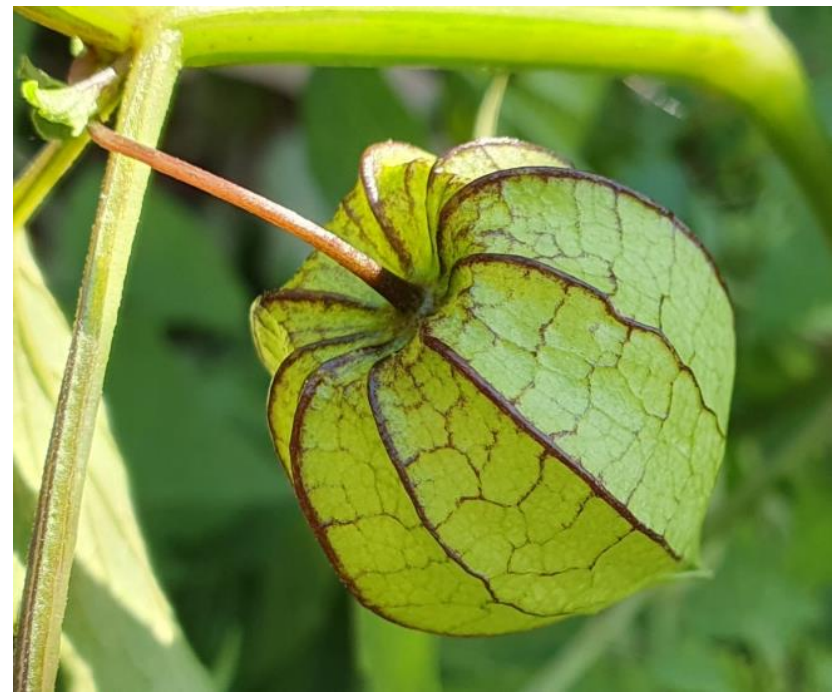

Figura 3: Cáliz fructífero

Figure 3: Fruiting calyx

Después de su detección en el noreste ibérico, la dinámica expansiva de $P$. angulata en otras regiones mediterráneas y su gran capacidad potencial para infestar cultivos de verano irrigados (Travlos, 2012; Ozaslan et al., 2017) hacen probable que en un futuro próximo colonice otras zonas de la Península (Fig. 2). En relación con las especies de Physalis previamente reconocidas en las síntesis florísticas ibéricas (Sanz \& Sobrino, 2012), P. angulata es morfológicamente próxima a $P$. ixocarpa, pero se distingue bien por varios caracteres (Waterfall, 1958, 1967; Hawkes, 
1972; Benítez de Rojas \& Magallanes, 1998; Sullivan, 2004; Bean, 2006; Ward, 2008; Landrum et al., 2013), en especial por la sección del caliz fructífero (circular en $P$. ixocarpa, angulosa en $P$. angulata, con 10 pliegues longitudinales) (Fig. 3) y por la longitud de los pedicelos (en la antesis hasta $5 \mathrm{~mm}$ en $P$. ixocarpa y $5-20 \mathrm{~mm}$ en $P$. angulata; en fruto hasta $15 \mathrm{~mm} P$. ixocarpa y hasta $40 \mathrm{~mm} P$. angulata). Un carácter adicional es el color de la corola, pero puede inducir a confusión, ya que siempre presenta manchas pardo-purpúreas marcadas en la garganta en $P$. ixocarpa, mientras que en $P$. angulata las manchas pueden faltar o bien existir pero ser bastante tenues (Fig. 4), como sucede a menudo en las poblaciones introducidas en Europa. $P$. angulata es también algo similar a $P$. philadelphica, pero de ésta se distingue más fácilmente porque presenta flores y frutos claramente más pequeños (corola $13-30 \mathrm{~mm}$ y fruto hasta $60 \mathrm{~mm}$ en $P$. philadelphica; corola y fruto hasta $10 \mathrm{~mm}$ en $P$. angulata).

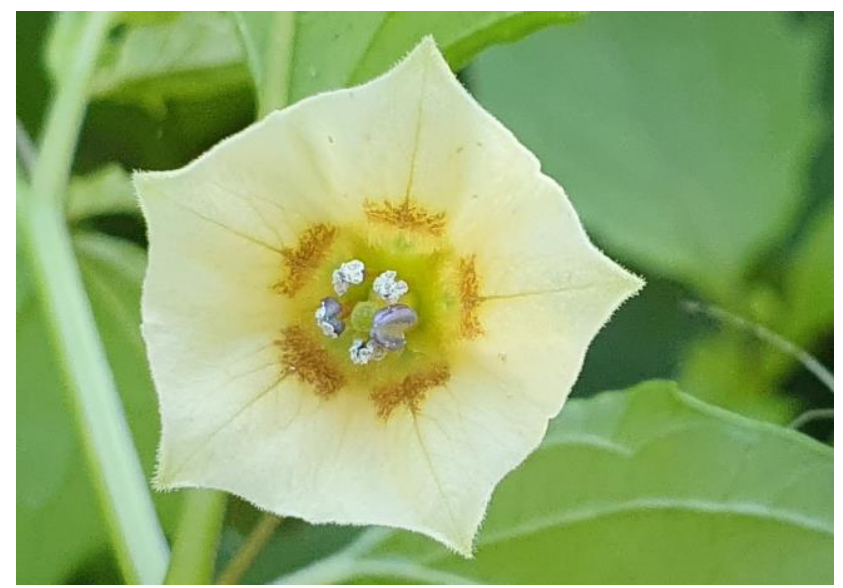

Figura 4: Flor

Figure 4: Flower

\section{Referencias}

Amaral Franco, J. \& Pinto da Silva, A.R. (1964). Plantas novas e novas áreas para a flora de Portugal. Agronomia Lusitana, 24(3), 194-195.

Aymerich, P. (2016). Contribució al coneixement de la flora al-lòctona del nord i centre de Catalunya. Orsis, 30, 11-40. doi: https://doi.org/10.5565/rev/orsis.26

Aymerich, P. \& Sáez, L. (2015). Comentaris i precisions previs a la Checklist de la flora de Catalunya (nord-est de la península Ibèrica). Orsis, 29, 1-68. doi: https://doi.org/10.5565/rev/orsis.18

Aymerich, P. \& Sáez, L. (2019). Checklist of the vascular alien flora of Catalonia (northeastern Iberian Peninsula, Spain). Mediterranean Botany, 40(2), 215-242.

doi: https://doi.org/10.5209/mbot.63608

Batard, R. (1970). Sur l'identification d'une espèce de Physalis subspontanée au Portugal. Boletim da Sociedade Broteriana, 44(2), 343-367.

Bean, A.R. (2006). Physalis (Solanaceae) in Australia - nomenclature and identification. Newsletter of Australian Systematic Botany Society, 127, 6-9.
Benítez de Rojas, C. \& Magallanes, A. (1998). El género Physalis (Solanaceae) de Venezuela. Acta Botanica Venezuelica, 21(2), 11-42.

Berselli, C., Bonali, F., Labadini, A. \& Marenzi, P. (2019). Segnalazioni floristiche per la provincia di Cremona. Pianura. Scienze e storia dell'ambiente padano, 38, 136-143.

Casasayas, T. (1989). La flora al-lòctona de Catalunya. Tesis doctoral. Universitat de Barcelona.

Deanna, R., Larter, M.D., Barboza, G.E. \& Simith, S.D. (2019). Repeated evolution of a morphological novelty: a phylogenetic analysis of the inflated fruiting calyx in the Physalidae tribe (Solanaceae). American Journal of Botany, 106(2), 270-279.

doi: https://doi.org/10.1002/ajb2.1242

Electronic Flora of South Australia (2020). Physalis angulata factsheet. Recurso electrónico en www.flora.sa.gov.au. Consulta realizada en 2010-2020.

Essl, F. \& Rabitsch, W. (2002). Neobiota in Österreich. Wien: Umweltbundesamt (Federal Envionment Agency-Austria).

Galasso, G. \& al. (2018). An updated checklist of the vascular flora alien to Italy. Plant Biosystems, 152(2), 179-303. https://doi.org/10.1080/11263504.2018.1441197

Gönen, O., Yildirim, A. \& Uygur, F.N.(2000). A new record for the flora of Turkey Physalis angulata $\mathrm{L}$. Turkish Journal of Botany, 24, 299-301.

Hawkes, J.G. (1972). Physalis. In: Tutin T.G. \& al. (Eds.), Flora Europaea, vol. 3. (p. 196). Cambridge, UK: Cambridge University Press.

Landrum, L.R., Barber, A., Barron, K., Coburn, F.S., Sanderford, K. \& Setaro, D. (2013). Vascular plants of Arizona: Solanaceae part four: Physalis L. and Quincula Raf. Ground Cherry, Tomatillo. Canotia, 9, 1-12.

Milović, M., Mitic, B. \& Alegro, A. (2010). New neophytes in the flora of Croatia. Natura Croatica, 19(2), 407-431.

Musarella, C.M., Stinca, A., Cano-Ortiz, A., Laface, V.L.A., Petreli, R., Esposito, A. \& Spampinato, G. (2020). New data on the alien vascular flora of Calabria (Southern Italy). Annali di Botanica, 10, 55-66.

doi: https://doi.org/10.13133/2239-3129/14838

Ozaslan, C., Farooq, S., Onen, H., Ozcan, S., Bukun, B. \& Gunal, H. (2017). Germination biology of two invasive Physalis species and implications for their management in arid and semi-arid regions. Scientific Reports, 7, 16960. doi: https://doi.org/10.1038/s41598-017-17169-5

Pysek, P., Danihelka, J., Sádlo, J., Chrtek, J., Chytry, M., Jarosík, C., Kaplan, Z., Krahulec, F., Moravcová, L., Pergl, J., Stajerová, K. \& Tichý, L. (2012). Catalogue of alien plants of the Czech Republic (2nd edition): checklist update, taxonomic diversity and invasion patterns. Preslia, 84, 155255.

Rodríguez, J., Deanna, R. \& Chiarini, F. (2020). Karyotype asymmetry shapes and diversity within the physaloids (Physalidinae, Physalidae, 
Solanaceae). Systematics and Biodiversity: en prensa.

doi: https://doi.org/10.1101/2020.04.21.053181

Sanz, M., \& Sobrino, E. (2012). Physalis L. In: S. Talavera, M. Arista, M., M.P. Fernández Piedra, M.J. Gallego, P.L. Ortiz, C. Romero Zarco, F.J. Salgueiro, S. Silvestre, S. \& A. Quintanar (eds.) Flora iberica. Vol XI. Gentianaceae-Boraginaceae: (pp: 204-209). Madrid: Real Jardín Botánico, CSIC.

Senar, R. \& Cardero, S. (2019). Dades de plantes al-lòctones per a l'est de la península Ibèrica. Collectanea Botanica, 38, 009. https://doi.org/10.3989/collectbot.2019.v38.009

Stace, C. (2010). New flora of the British Isles. Third Edition. Cambridge, UK: Cambridge University Press.

Stešević, D. \& Bubanja, N. (2017). Five new alien species in the flora of Montenegro: Coreopsis tinctoris Nutt., Ipomoea indica (Burm.) Merr., Lupinus $x$ regalis Bergmans, Physalis angulata L. and Solidago canadensis L., and new possible threats to the biodiversity. Natura Croatica, 76(1), 98-102. https://doi.org/10.1515/botcro-2016-0048

Sullivan, J.R. (2004). The genus Physalis (Solanaceae) in the southeastern United States. Rhodora, 106(928), 305-326.

Tison, J.M. \& Foucault, B. (2014). Flora Gallica - Flore de France. Mèze, France: Biotope éditions.

Tison, J.M., Jauzein, P. \& Michaud, H. (2014). Flore de la France méditerranéenne continentale. Conservatoire Botanique National Méditerranéen de Porquerolles. Turriers, France: Naturalia Publications.

Travlos, I.S., Economou, G. \& Lyberopoulou, S. (2010). The weed Physalis angulata in western Greece. Proceedings of $16^{\text {th }}$ Conference of the Greek Weed Science Society. (p. 41). Karditsa, 12 December.

Travlos, I.S. (2012). Invasiveness of cut-leaf groundcherry (Physalis angulata L.) populations and impact of soil watre and nutrient availability. Chilean Journal of Agricultural Research, 72(3),
358-363. doi: http://dx.doi.org/10.4067/S071858392012000300009

Travlos, I.S. (2013). Datasheet: Physalis angualata (cutleaf grouncherry). CABI-Invasive Species Compendium. Recurso electrónico en www.cabi.org. Consulta realizada en 20-10-2020

Vargas-Ponce, O., Sánchez, J., Zamora, M.P. \& Valdivia, L.E. (2016). Traditional management of a small-scale crop of Physalis angulata in Western Mexico. Genetic Resources and Crop Evolution, 63, 1383-1395. doi: https://doi.org/10.1007/s10722-015-0326-3

Vázquez, M., Martín, A., Ramos, S., Doncel, E., Blanco, J. \& Lucas, A.B. (2002). Aportaciones a la flora de Extremadura (España). Acta Botanica Malacitana, 27, 259-261.

Verloove, F. (2003). Physalis ixocarpa Brot. ex Hornem. and Verbena litoralis Kunth, new Spanish xenophytes and records of other interesting alien vascular plants in Catalonia (Spain). Lazaroa, 24, 7-11.

Verloove, F. (2006). Catalogue of neophytes in Belgium (1800-2005). Scripta Botanica Belgica 39, 1-89.

Ward, D.B. (2008). Keys to the flora of Florida: 19, Physalis (Solanaceae). Phytologia, 90(2), 198207.

Waterfall, U.T. (1958). A taxonomic study of the genus Physalis in North America north of Mexico. Rhodora, 60, 107-114, 128-142, 152-173.

Waterfall, U.T. (1967). Physalis in Mexico, Central America and the West Indies. Rhodora, 69, 82120, 202-239, 319-329.

Whitson, M. \& Manos, P.S. (2005). Untangling physalis (Solanaceae) from the Physaloids: A twogene phylogeny of the Physalinae. Systematic Botany, 30(1), 216-230. doi: https://doi.org/10.1600/0363644053661841

Zamora-Tavares, M.P., Martínez, M., Magallón, S., Guzmán-Dávalos, L. \& Vargas-Ponce, O. (2016). Physalis and physaloids: A recent and complex evolutionary history. Molecular Phylogenetics and Evolution, 100, 41-50. doi: https://doi.org/10.1016/j.ympev.2016.03.032 